\title{
The Interdisciplinary Education Perception Scale (IEPS): which factor structure?
}

\author{
Brett Vaughan $^{1,3,4}$, Chris Macfarlane ${ }^{1}$, Terri Dentry ${ }^{2,3}$, Gina Mendoza ${ }^{2,3}$ \\ ${ }^{1}$ College of Health \& Biomedicine, Victoria University, Melbourne, Australia. ${ }^{2}$ College of Exercise \& Sports \\ Science, Victoria University, Melbourne, Australia. ${ }^{3}$ Institute of Sport, Exercise and Active Living, Victoria \\ University, Melbourne, Australia. ${ }^{4}$ School of Health \& Human Sciences, Southern Cross University, Lismore, \\ Australia.
}

\section{ARTICLE INFO}

Received

Accepted

Published

$15 / 03 / 2014$

: 27/07/2014

: 01/09/2014

\section{KEYWORD}

Interdisciplinary

Interprofessional

collaboration

Interprofessional learning

Psychometrics

Factor analysis

Reliability

Validity

\section{ABSTRACT}

Introduction: The Interdisciplinary Education Perception Scale (IEPS) is used to assess the perception of one's own profession and the relationship of that profession to others. A number of models of the IEPS exist within the literature. Objective: The aim of the present study was to explore the most appropriate factor structure of the IEPS in an Australian health profession student and clinical educator population. Method: The IEPS was completed online. Confirmatory factor analysis (CFA) using the Maximum Likelihood Estimation method was employed for the analysis. The data were entered into each of the 4 IEPS models identified in the literature. Result: Data did not adequately fit any of the models identified in the literature. Two of the models described in the literature appeared to be stronger than that proposed by the original IEPS authors. Conclusion: Further research using both CFA and item response theory is required to improve the psychometrics of the IEPS.

(c) Medical Education Department, School of Medical Sciences, Universiti Sains Malaysia. All rights reserved.

CORRESPONDING AUTHOR: Dr Brett Vaughan, College of Health \& Biomedicine, Flinders Lane Campus, Victoria University, PO Box 14428, Melbourne, Victoria, 8001, Australia.Email: brett.vaughan@vu.edu.au

\section{Introduction}

There are a range of questionnaires that have been developed to assess interprofessional (IP) education before, during and after teaching and clinical education programs. Thannhauser et al. (1) undertook a systematic search of the literature and identified 23 measures. These authors reported that the majority of the measures have only been used once, with very few papers reporting the psychometric properties. Of the 23 measures identified, the 2 most widely used are the Readiness for Interprofessional Learning Scale (RIPLS) and the Interdisciplinary Education Perception Scale (IEPS). The IEPS is considered to be a measure of attitudes and perceptions of the interprofessional experience and the literature contains a number of versions of the IEPS.

Luchet et al. (2) produced the original version of the IEPS for use as a self-report measure in a health professional student population. In the development of the IEPS, 143 students were recruited. The scale comprised 18 -items divided into 4 subscales: Competency and autonomy; Perceived need for cooperation; Perception of actual cooperation; and Understanding others' 
value. McFadyen et al. (3) reported concerns with the psychometric properties of the IEPS, particularly the test-retest reliability and the subscale structure. Work by these authors with Scottish health science students proposed a 12item, 3-subscale version of the IEPS. The three subscales were: Competence and autonomy; Perceived need for cooperation; and Perception of actual cooperation. An area of concern is the Perceived need for cooperation subscale as both McFayden et al. (3) and Leitch (4) both demonstrated low reliability.

Williams and Webb (5), using an exploratory factor analysis with principal axis factoring, developed a 12-item, 2 factor structure for the IEPS in an Australian paramedic student population. The factors were labelled Cooperation and teamwork, and Positivity. Leitch (4) examined the structure of the IEPS in a graduate health student population using a confirmatory factor analysis (CFA) approach. Initial model fit to the IEPS structure identified by Luecht et al. (2) was reported as poor and modifications to the model did not improve the fit. The fit indices for the model proposed by McFayden et al. (3) were then examined and demonstrated an acceptable model fit. A 16 item, 3-subscale measure was developed through a post-hoc review of the literature in order to identify factors that may impact on interprofessional collaboration. Competence and autonomy, Cooperation; and Prestige were the 3 subscales. Leitch (4) suggested the Prestige subscale is a strength of the alternate scale, particularly compared to that proposed by McFayden et al. (3).

In order to continue developing the literature around the construct validity of the IEPS, the present study employed a confirmatory factor analysis (CFA) approach to identify a model from the literature that fit the data. This approach has not previously been used to investigate the factor structure of the IEPS. CFA is generally used where the factors, and items within the factors, have been established through an exploratory factor analysis or previous CFA $(6,7)$. As suggested by Levine et al. (8) validity of a scale in one population or study does not necessarily translate to other populations or studies. As such, investigating the factor structure of published scales is imperative and provides further validity evidence for that scale (8). Given the multiple versions of the IEPS available in the literature, a CFA was undertaken to investigate the most appropriate factor structure in an Australian allied health student and clinical educator population.

\section{Method}

Data were collected as part of a larger study into interprofessional education at Victoria University (VU) and Southern Cross University (SCU). Students enrolled in the health professions programs at these two universities, and clinical educators involved in the education of these students were invited to complete an online version of the IEPS (in addition to a demographic questionnaire and the RIPLS). The IEPS items were taken from the scale developed by Luecht et al. (2). The questionnaires were placed online using the Qualtrics program (Qualtrics, USA). The demographic data and RIPLS data are not presented here.

Potential participants received an email from the authors or another researcher (who was part of the larger study) inviting them to participate. The email contained a web link to the questionnaire. Consent to participate was implied by completing the questionnaire and all responses were anonymous. This study was approved by the VU and SCU Human Research Ethics Committees.

Data were exported from Qualtrics to SPSS Version 21 (IBM Corp, USA). Assessment of missing data and normality testing were undertaken in SPSS. AMOS Version 21 (IBM Corp, USA) was used for the CFA. Each of the four models to be assessed was established in the program and examined independently using the same data set. Maximum Likelihood Estimation was used for the analysis with a variety of fit indices generated (Table 1). No modifications to the models were made during the analysis. 
Table 1. Model fit statistics.

\begin{tabular}{|c|c|c|c|c|c|c|c|c|}
\hline & $x^{2}$ & $\chi^{2} / \mathrm{DF}$ & TLI & RMR & NFI & RFI & CFI & RMSEA $(95 \% \mathrm{Cl})$ \\
\hline Luecht et al. & 390.61 & 3.03 & 0.68 & 0.009 & 0.65 & 0.59 & 0.73 & $\begin{array}{c}0.113(0.100- \\
0.125)\end{array}$ \\
\hline McFayden et al. & 116.38 & 2.28 & 0.86 & 0.005 & 0.83 & 0.78 & 0.89 & $\begin{array}{c}0.090(0.068- \\
0.111)\end{array}$ \\
\hline Williams et al. & 168.86 & 3.18 & 0.76 & 0.006 & 0.75 & 0.69 & 0.81 & $\begin{array}{c}0.117(0.097- \\
0.137)\end{array}$ \\
\hline Leitch & 213.86 & 2.11 & 0.83 & 0.006 & 0.76 & 0.72 & 0.86 & $\begin{array}{c}0.084(0.068- \\
0.099)\end{array}$ \\
\hline Good model fit & & $2.0-3.0$ & $>0.90$ & $<0.05$ & $>0.95$ & $>0.95$ & $>0.95$ & $0.05-0.08$ \\
\hline
\end{tabular}

Legend

DF - Degrees of freedom, TLI - Tucker-Lewis Index, RMR - Root mean square residual, NFI - Normed fit index, RFI -

Relative fit index, $\mathrm{CFI}$ - Comparative fit index, RMSEA - Root mean square error of approximation

\section{Result}

Responses were received from 184 participants. Data were missing from 23 respondents and subsequently deleted. Each item was then transformed using a square-root normalisation. Data from 161 respondents (130 students, 31 clinical educators) were entered into the 4 IEPS models. The results of each CFA are presented in Table 1. The fit for all models did not achieve the accepted model fit levels for each of the statistics presented. Each model demonstrated a combination of both relatively strong and weak fit statistics.

\section{Discussion}

The current paper has presented a CFA of four models of the IEPS that have previously been presented in the literature. No one model consistently demonstrated fit statistics at or above the recommended levels suggesting measurement invariance or misfit due to chance (8). Bryne and Watkins (9) provide some ideas as to why this measure invariance or construct invalidity may exist: different respondent groups; differences in the meaning of items between populations; and method of measurement. In the present study it is likely that the different groups and different meanings could account for the results.
The statistics for the models proposed by McFayden et al. (3) and Leitch (4) appear to be the closest fit for the IEPS using the present data set. However, both models would require further modification to fit the data in the present study. Each of the models by McFayden et al. (3) and Leitch (4) have their own advantages and disadvantages. The large sample size and number of health professions involved in the development of the MacFayden et al. (3) improves the generalisability however as these authors, along with Leitch (4), have suggested, only two items on the Perceived need for cooperation subscale may be problematic. The sample size in the Leitch (4) model means the scale is potentially more generalisable, however further research into this model is required in undergraduate student populations. Lack of data fit to the Williams and Webb (5) model may be the result of a substantial reduction in scale items and use of a single health profession population.

Leitch (4) demonstrated poor data fit with the original IEPS factor structure proposed by Luecht et al. (2) (TLI $=0.68$, CFI $=0.73$, RMSEA = 0.13). These results are comparable with the present study, suggesting that the original IEPS factor structure may not be appropriate, and supports the position of Levine et al. (8) who suggest scales should be subjected 
to further investigation to develop their construct validity. Although each of the IEPS models could have been modified to fit the data obtained in the present study, this was not performed as authors have previously argued that this constitutes an exploratory, rather than confirmatory, factor analysis approach (10). It would be reasonable to perform an exploratory factor analysis where a CFA demonstrates poor model fit (11). Given the lack of model fit, it is suggested that further research be undertaken using item response theory in order to establish a version of the IEPS that will be sample independent rather than the sample dependent measure produced by classical test theory statistics. Rasch analysis would be appropriate to analyse the IEPS however this was not undertaken in the present study due to the small sample size. This will be undertaken in future research.

A potential issue with the current study is the relatively small sample size for a CFA, although the ratio of respondents to items was greater than the minimum of 5:1 suggested by Di Stefano and Hess (7), and greater than the 100 suggested by Kline (12). Statistical techniques were employed to overcome the non-normality of the data however it may have impacted on the model fit. As with any self-report measure, a response and non-response bias may be present (5) in that respondents who are more open to IPE may be more likely to respond to the invitation to complete the questionnaire. Future studies should investigate the fit of the data to one of the four models in order to improve the validity of the scale, and continue to develop a profile of the psychometric properties of the IEPS.

\section{Conclusion}

Given the variety of the results for the fit statistics for each of the models presented in the current study, further analysis of the psychometric properties of the IEPS is required. Each of the models analysed had its strengths and weakness. In particular, it would appear that the structure proposed by the original authors of the IEPS is not appropriate. The present study did not attempt to modify a model to fit the data.
However, it is likely that another factor structure would have been identified. Given these equivocal results, it is recommended that the construct validity of the IEPS be investigated further using item response theory.

\section{References}

1. Thannhauser J, Russell-Mayhew S, Scott C. Measures of interprofessional education and collaboration. J Interprof Care. 2010;24:33649.

2. Luecht R, Madsen M, Taugher M, Petterson B. Assessing professional perceptions: design and validation of an Interdisciplinary Education Perception Scale. J Allied Health. 1990;19:181.

3. McFadyen A, Maclaren W, Webster V. The Interdisciplinary Education Perception Scale (IEPS): An alternative remodelled sub-scale structure and its reliability. J Interprof Care. 2007;21:433-43.

4. Leitch J. Exploring psychometric properties of the interdisciplinary education perception scale in health graduate students. J Interprof Care. 2013:1-6.

5. Williams B, Webb V. Examining the measurement properties of the Interdisciplinary Education Perception Scale (IEPS) in paramedic education. Nurse Educ Today. 2012;33:981-5.

6. Henson RK, Roberts JK. Use of exploratory factor analysis in published research common errors and some comment on improved practice. Educ Psychol Measure. 2006;66:393-416.

7. DiStefano C, Hess B. Using confirmatory factor analysis for construct validation: An empirical review. J Psychoeduc Assess. 2005;23:225-41.

8. Levine T, Hullett CR, Turner MM, Lapinski MK. The desirability of using confirmatory factor analysis on published scales. Communi Res Reports. 2006;23:309-14.

9. Byrne BM, Watkins D. The issue of measurement invariance revisited. J CrossCultural Psychol. 2003;34:155-75.

10. Bollen KA. Structural models with latent variables. New York, USA: Wiley; 1989. 
11. Schmitt TA. Current methodological considerations in exploratory and confirmatory factor analysis. J Psychoeduc Assess. 2011;29:304-21.

12. Kline RB. Principles and practice of structural equation modeling: Guilford press; 2011. 